\title{
A Low-Loss MEMS Tunable Capacitor with Movable Dielectric
}

\author{
Y. Zhu, M. R. Yuce, S. O. R. Moheimani \\ School of Electrical Engineering and Computer Science, the University of Newcastle \\ University Drive, NSW, 2308, Australia \\ Email: \{yong.zhu, mehmet.yuce, reza.moheimani\}@newcastle.edu.au
}

\begin{abstract}
This paper reports a MEMS tunable capacitor with a new actuation principle. The new design adopts electrostatic actuation of an electrically floating movable dielectric. This enables us to achieve a high $Q$ factor by eliminating the loss associated with springs in the RF signal path. Also, the design can achieve a high tuning range, by using additional actuation combs and thus eliminating the pull-in effect. Since no DC bias is applied on the plates of variable capacitor, the parallel plates 1/3 gap limitation does not apply. The designed devices were fabricated in a SOI MEMS process, with a $25 \mu \mathrm{m}$ thick device layer and minimum gap of $2 \mu \mathrm{m}$. Measurement results show that the tunable capacitor has a $135 \mathrm{fF}$ initial capacitance with a tuning range of $367 \%$ and a $Q$ factor of 56 at $1 \mathrm{GHz}$ by bidirectional actuation.
\end{abstract}

\section{INTRODUCTION}

RF tunable capacitors are key building blocks in many wireless communication applications, such as voltage controlled oscillators (VCO), tunable filters and phase shifters $[1,2]$. These devices typically require a wide tuning range and high quality factor. Compared with semiconductor varactors, MEMS tunable capacitors have the potential for an extended tuning range, higher linearity and lower loss, along with the reduction in weight, size, and cost of communication systems [3, 4]. However, traditional MEMS capacitor designs clearly exhibit a $\mathrm{Q}$ factor versus actuation voltage trade-off: the $\mathrm{Q}$ factor of micromechanical tunable capacitor is limited by resistive losses in the suspension beams, which are often made narrow and long to make it soft for low voltage actuation [5]. A method of moving electrically floating plate has been proposed to provide a much better Q factor by eliminating the inevitable spring resistance in the RF signal path [6]. However, the tuning ratio is relatively low $(41 \%)$ because of the pull-in effect [6]. The pull-in instability, due to which only $33 \%$ of the gap between plates could be covered smoothly, greatly reduces the useful dynamic range of MEMS tunable capacitors.

In this paper, a novel MEMS tunable capacitor with floating plate is introduced. By adding a comb actuator instead of applying DC bias on parallel plate in the RF signal path in [6], the pull-in effect is eliminated. The test results show that the tunable capacitor has a high tuning range of $367 \%$ and a $\mathrm{Q}$ factor of 56 at $1 \mathrm{GHz}$.

\section{DEVICE DESIGN}

\section{A. Tuning range}

Tuning range is the ratio of variable capacitor's maximum changed capacitance to the minimum capacitance, defined as:

$$
\text { Tuning range }=\frac{C_{\max }-C_{\min }}{C_{\min }}
$$

To increase the tuning range, one must maximize $C_{\max }{ }^{-}$ $C_{\text {min }}$. However, in a parallel plate capacitor, the electrostatic instability known as pull-in phenomenon limits the tuning range of the capacitor to a theoretical maximum of $50 \%$ [3].

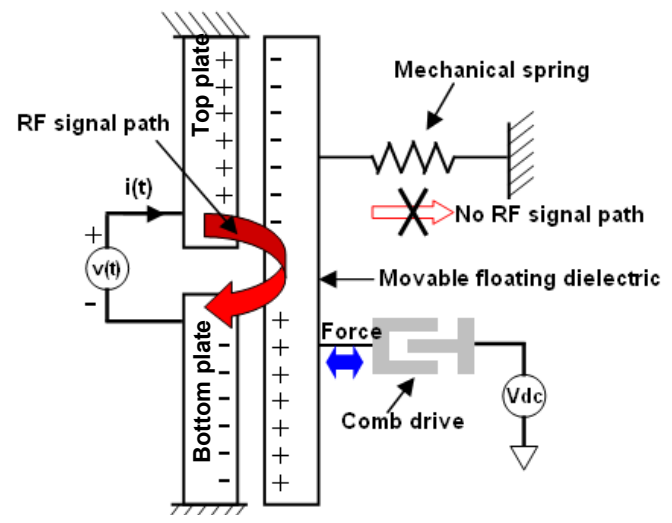

Fig. 1: Conceptual schematic view of the proposed tunable capacitor with an electrically floating dielectric.

Figure 1 presents the conceptual schematic view of the tunable capacitor design, identifying key components and the RF signal path. In our design, an additionally comb actuator
This research is funded by Australian Research Council (ARC) discovery grant- DP0774287 
is introduced in the device to make the actuation and the RF signal paths separate, as shown in Figure 1.

The gap between the dielectric and the plates can be adjusted by a DC voltage on drive combs, making the capacitor tunable over large range without being effected by the pull-in mechanism. The tunable capacitor is actuated laterally by comb drives on both sides. Comb drive actuation allows for the actuation force on the tunable capacitor to be approximately independent of displacement, which means the capacitor gap can be theoretically zero (infinite capacitance). To get a wider dynamic range than a commonly designed one direction actuated tunable capacitor, a bidirectional design has been adopted with two sets of combs, as shown in Figure 2. When a DC voltage is applied to the left comb drive, the tunable capacitor value becomes higher. Conversely, when a DC voltage is applied on the right comb drive, the capacitance decreases.

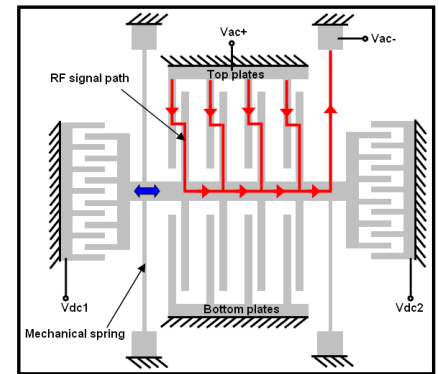

(a)

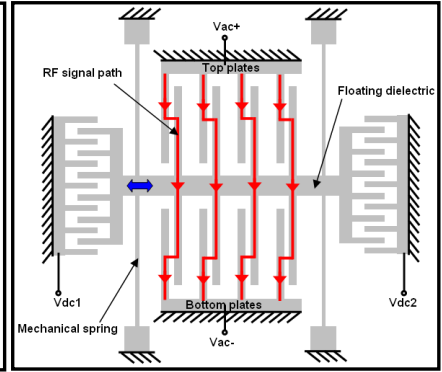

(b)
Fig. 2: Schematic views of tunable capacitor design with RF signal through spring (a) and dielectric (b).

\section{B. Qfactor}

The quality factor is a measure of the energy loss in a microwave circuit. It is defined as the ratio of the energy stored in a device to the energy dissipated in it per cycle. A discrete capacitor can be modeled as a series $L_{s}, C_{s}, R_{s}$ model, and suffers from a self-resonant frequency $f_{s}$, and $f_{s}=1 /\left(2 \pi \sqrt{L_{s} C_{s}}\right)$. Below this frequency, the capacitor $\mathrm{Q}$ factor is dominated by the series capacitance $C_{s}$, and is defined as [7]

$$
Q=\frac{1}{\omega C_{s} R_{s}}
$$

$R_{s}$ includes all series resistance in the RF signal path. To achieve a high Q factor, the series resistance must be reduced. In traditional MEMS tunable capacitor designs, the series resistance in the suspension beams dominated the device quality factor, because the beams are often made narrow and long to make them less stiff for a low voltage actuation.

Figure 2 illustrates a movable electrically floating dielectric suspended beside the top and the bottom plates of a capacitor by mechanical springs. Unlike the traditional designs [6], in our prototype, the RF signal does not pass through springs. Thus, a better $Q$ can be achieved by eliminating the high resistive loss in the springs.

Schematic views of the actual structure design with different test methods for comparison purpose are given in Figure 2. Figure 2(a) shows traditional designs, where the RF signal passes through spring from the top plates to the anchor, causing high loss in the spring. In our design, as shown in Figure 2(b), the RF signal flows through the floating dielectric from the top plates to the bottom plates, avoiding the loss in beams to achieve a high quality factor. The key design parameters for the tunable capacitor device are presented in Table I.

TABLE I.

DESIGN PARAMETERS FOR TUNABLE CAPACITOR

\begin{tabular}{cc}
\hline Parameter & Value \\
\hline Comb drive gap $(\mu \mathrm{m})$ & 3 \\
Tunable capacitor initial gap $(\mu \mathrm{m})$ & 3 \\
Initial space between two tunable capacitors $(\mu \mathrm{m})$ & 9 \\
Tunable capacitor length $(\mu \mathrm{m})$ & 295 \\
Tunable capacitor electrode width $(\mu \mathrm{m})$ & 8 \\
Number of tunable capacitor each side & 27 \\
Beams length $(\mu \mathrm{m})$ & 295 \\
Beams width $(\mu \mathrm{m})$ & 4 \\
Structures thickness $(\mu \mathrm{m})$ & 25 \\
\hline
\end{tabular}

\section{FABRICATION}

The device was fabricated in a commercial Silicon-OnInsulator (SOI) MEMS foundry (MEMSCAP) process [8] with a $25 \mu \mathrm{m}$ thick device layer and minimum gap of $2 \mu \mathrm{m}$. Figure 3 provides an illustrated summary of this process:

1) Surface metal pads are patterned on a highly doped ntype $25 \mu \mathrm{m}$ silicon device layer to allow for ohmic contact.

2) Deep reactive ion etch (DRIE) from the front side of the
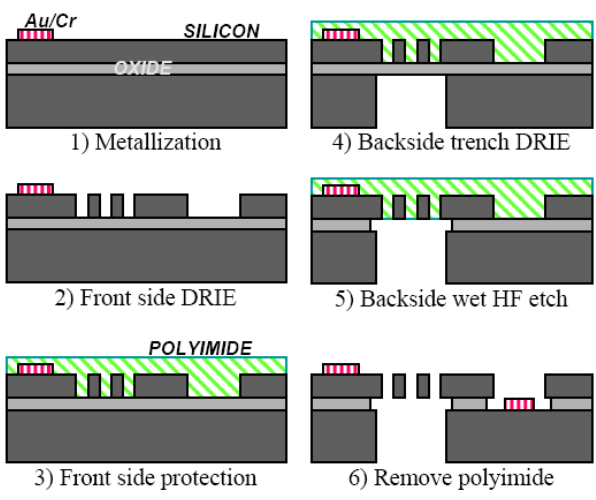

Fig. 3: SOI fabrication process through MEMSCAP 
wafer to define both the anchored and movable features of the structure.

3) A protective polyimide layer is applied to the front side.

4) A deep trench underneath the movable structures is created by etching through the substrate using DRIE.

5) The exposed buried oxide is removed using a wet HF etch.

6) The polyimide coat on the front side is removed by oxygen plasma, thereby allowing the movable structure to be fully released. Then a large contact metal pad is patterned on the substrate for electrical contact.

The image of the whole device and a section of it taken under a Scanning Electron Microscope (SEM) are provided in Figure 4.

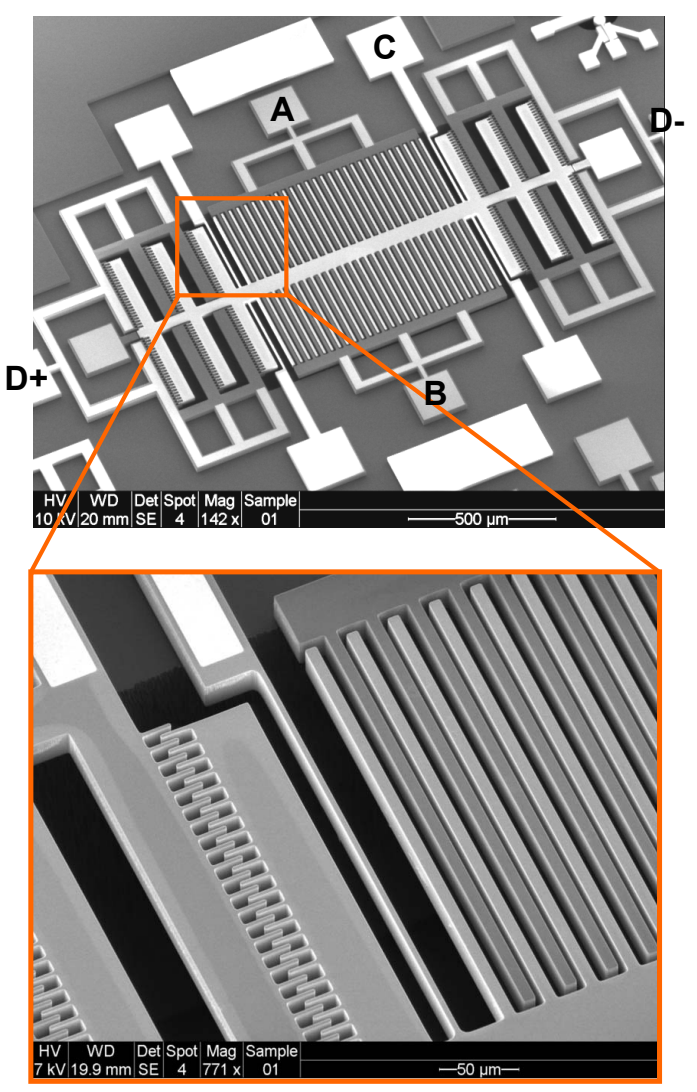

Fig. 4: SEM image of the micromachined tunable capacitor.

\section{EXPERIMENTAL RESULTS}

A SUSS PM5 Analytical Probe Station was utilized for the initial chips test. The SOI chip was fixed on the surface of the grounded platform by vacuum section, and then probes with cables were pressed on the device pads for the electrical connection of the device and external instruments. The tunable capacitor was measured using Agilent E4980A precision LCR meter. DC tuning voltages were applied to the actuators and the capacitance values were recorded at $2 \mathrm{MHz}$. For comparison, we conducted two sets of measurements using the same tunable capacitor, one for each arrangement shown in Figure 2.

\section{A. Traditional design measurement}

In Figure 2 (a), to measure the capacitance and the $\mathrm{Q}$ factor of the traditional design, one probe was put on the pad of a beam's anchor ( $\mathrm{C}$ in Figure 4), and the other probe was pressed on the pad of a fixed comb plate (A in Figure 4). Then, DC voltages of $0-120 \mathrm{~V}$ were applied to the two actuator pads ( $\mathrm{D}+$ and $\mathrm{D}$ - in Figure 4) in sequence to achieve bidirectional actuation. The capacitance change vs. actuation voltages are shown in Figure 5. The capacitance varied from $764 \mathrm{fF}$ to $2075 \mathrm{fF}$ with the voltage range of $-120 \mathrm{~V}$ to $120 \mathrm{~V}$, suggesting a tuning range of $172 \%$. The initial capacitance without applied voltage is $830 \mathrm{fF}$.

To determine the quality factor, the equivalent series resistance was calculated by using tunable capacitor's dimensions and measured sheet resistance. The sheet resistance was measured to be $14.8 \mathrm{ohm} /$ square. Thus, the Q factor is calculated as 0.35 at $1 \mathrm{GHz}$ using equation (2).

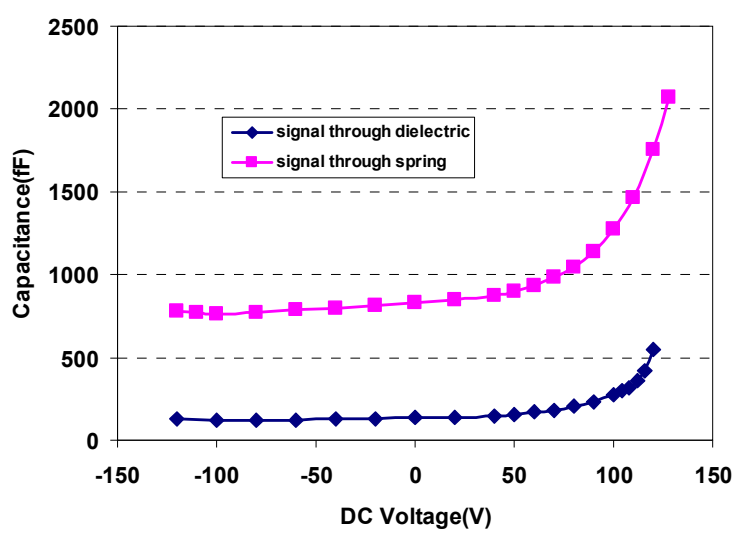

Fig. 5: Measured capacitor changes vs. applied dc bias

\section{B. Floating dielectric design}

To measure the $\mathrm{C}-\mathrm{V}$ tuning curve and $\mathrm{Q}$ factor of the floating dielectric capacitor, LCR meter's probes were connected to A and B pads, while pad $\mathrm{C}$ was left electrically floating. Then, a $0-120 \mathrm{~V}$ DC voltage was applied to $\mathrm{D}+$ and D- pads for the actuation. A plot of capacitance change vs. actuation voltages is shown in Figure 5. The capacitance changed from $118 \mathrm{fF}$ to $551 \mathrm{fF}$, suggesting a tuning range of $367 \%$. The initial capacitance without an applied voltage is $135 \mathrm{fF}$, which is smaller than the case associated with the traditional design measurement. There are two main reasons: the tunable capacitor's gap is twice wider than the traditional design; and the parasitic capacitance is much small because of no direct substrate silicon between pads $\mathrm{A}$ and $\mathrm{B}$.

The Q factor is calculated as 56 at $1 \mathrm{GHz}$ using the sheet resistance and tunable capacitor's dimensions. Q is much 
lower than 0.35 in the traditional case because of avoiding high loss in spring.

Note that the actuation voltage is high for most applications in RF circuits. This issue can be addressed easily by making the beams less stiff. Because of no $Q$ factor versus actuation voltage trade-off in [5], the beams can be made as narrow as required. For instance, the actuation voltage can be reduced to $15 \mathrm{~V}$ (1/8 of present maximum voltage) by halving the beams width to $2 \mu \mathrm{m}$, without incurring more resistive loss.

\section{CONCLUSION}

A novel MEMS tunable capacitor actuated with electrically floating silicon dielectric has been designed and characterized in this paper. By avoiding the resistive loss in suspended springs, the proposed tunable capacitor achieved a better $\mathrm{Q}$ factor than the traditional designs. The quality factor can be future increased further by changing the structure material silicon to metal, making the resistive loss less. These tunable capacitors are expected to find applications in low-loss $\mathrm{RF}$ applications such as VCO, filters and phase shifters.

\section{ACKNOWLEDGMENT}

The authors would like to thank Dave Phelan from the school of Medical Sciences at University of Newcastle for his help on SEM images.

\section{REFERENCES}

[1] Th. G. S. M. Rijks, J. T. M. Beek, P. G. Steeneken, M. J. E. Ulenaers, J. D. Coster and R. Puers, "RF MEMS Tunable Capacitors with Large Tuning Ratio", IEEE MEMS Conference, p. 777-780, 2004.

[2] A. Dec and K. Suyama, "Micromachined electromechanically tunable capacitors and their applications to RF IC's", IEEE Trans. Microwave Theory and Techn. 46(12), 1998, 2587

[3] H. A. C. Tilmans, W. D. Raedt and E. Beyne, "MEMS for Wireless communications: from RF-MEMS Components to RF-MEMS-SiP", J.Micromech.Microeng., vol. 13, p. S139-S163, 2003.

[4] M. R. Zadeh, A. K. Samarao, P. Monajemi, and F. Ayazi, "Lowvoltage large value tunable capacitors using self-aligned HARPSS", Proc. 21st IEEE International Conference on Micro Electro Mechanical Systems (MEMS 2008), Tucson, AZ, Jan. 2008, pp. 319322

[5] J. B. Yoon, C. T. C. Nguyen, "A High-Q Tunable Micromechanical Capacitor with Movable Dielectric for RF Applicaitons", IEEE IEDM, p. 489-492, 2000.

[6] Y. J. Yoon, H. S. Lee and J. B.Yoon, "MEMS Variable Capacitor Actuated with an Electrically Floating Plate", IEEE IEDM, p. 431-434, 2007.

[7] G. M. Rebeiz, "RF MEMS Theory, Design, and Technology", 1st edition, Wiley-Interscience, p328, 2002.

[8] http://www.memscap.com/en_mumps.html 